\title{
Path generation and navigation system design under ocean currents for an unmanned underwater vehicle
}

\author{
Kihun Kim ${ }^{* 1}$, Hyun-Taek Choi ${ }^{* 1}$, Sea-Moon Kim ${ }^{* 1}$, Chong-Moo Lee ${ }^{* 1}$ and Pan-Mook Lee ${ }^{* 1}$ \\ *1 Korea Ocean Research \& Development Institute \\ Jang-Dong 171, Youseong-Gu, Daejeon, Korea
}

\begin{abstract}
:
This paper describes path generation and navigation system design methodology under ocean currents using multiple sensors fusion. By adopting a natural cubic spline curve generation scheme, smooth path planning can be conducted considering major ocean current direction in heuristic manner. A multiple sensors based precise navigation scheme enables underwater vehicles to be controlled with qualified navigation information such as update rate, accuracy, precision.
\end{abstract}

\section{INTRODUCTION}

Path planning and precise navigation is essential issue to implement underwater robotic missions.

The path generation scheme can be evaluated by its optimality. For optimal path finding, there are well known schemes such as $A^{*}$, D*, potential field, genetic algorithms considering start position, goal position, obstacles and environments. But they are not practical enough to be applied in real sea operation for underwater vehicles. In this work, a human supervised path generation scheme considering known obstacles and environments such as ocean currents is suggested. To implement heuristic path generation scheme, a "natural" piecewise cubic spline scheme developed from computer aided design is introduced. Human supervision can be applied by just selecting nodal way-points, considering obstacle and current direction to construct naturally looking good sub-optimal path. The applied centripetal scheme designed by Eugene Lee (1989), can automatically generate piecewise natural cubic spline curves.

Another important issue is on the robust and precise underwater navigation implementation. The performance of a navigation sensor or kinds of algorithms can be evaluated in the view of three aspects: accuracy, precision and update rate. The accuracy means how much the estimated values coincide with the real values. The precision means how finely the resolution can be executed. The update rate means how fast the estimation value can be provided. The accuracy is especially important in wide area survey while the precision and update rate are essential in feedback control for attitude and waypoint tracking. Concerning underwater navigation, the submerged body can be described by 6 DOF motion (surge, sway, heave, roll, pitch, yaw) and altitude in addition. Usually attitudes, depth and altitude can be measured quite precisely with sufficient update rate by commercial sensors such as FOG-AHRS (Fiber optic gyro based attitude heading reference system, $100 \mathrm{~Hz}$ ), pressure sensor $(20 \mathrm{~Hz})$, and DVL $(5 \mathrm{~Hz})$. To achieve precise full state navigation information, the horizontal positions (north and east in navigation frame) should be examined with some efforts. Sensors which measure horizontal motions can be divided into two groups. One group measures relative motions to the navigation frame fixed on the earth such as inertial motion unit (acceleration and angular velocity) and Doppler velocity log (velocity). They show good performances in terms of precision and update rate. The other group measures absolute positions such as acoustic positioning systems (USBL, LBL, SBL). They have merits in point of accuracy. Multiple sensor fusion can be a good solution to achieve an accurate and precise performance with sufficient update rate by fusing their physical sensor characteristics.

Much research has been conducted to enhance the navigation performance in the horizontal plane. Whitcomb et al (1999) proposed a Doppler based navigation and investigated that the bottom rock navigation error is mainly due to heading estimation error and needs in-situ calibration. Kinsey and Whitcomb (2007) enhanced the in-situ calibration technique by comparing the absolute position and standalone dead-reckoning results

In this paper, the authors have adopted an improved fast covariance intersection method for multiple sensor data fusion which was proposed by Fränken and Hüpper (2005). This fusion scheme is chosen because its merits are robust, fast, easy to implement in online.

For outlier rejection of acoustically measured position data smoothing, Cleveland (1979) proposed a robust locally weighted regression and smoothing scheme. In this paper, the outlier rejection algorithm has been designed and executed concurrently with the multi-sensor data fusion algorithm.

Fig. 1 depicts the sensor fusion based navigation strategy adopted in this paper. It can be divided into three parts. The first part is a standalone DR algorithm enhancement. The second part is the outlier rejection algorithm and the third part is on the fusion of possible estimation candidates.

\section{GUIDANCE ALGORITHM CONSIDERING UNDERWATER CURRENTS}

To generate a practical guidance path for an underwater vehicle experiencing considerable ocean currents, the final approach angle near a goal should coincide with major current axis, i.e., the vehicle's desired heading angle should be chosen against the major current axis angle. 
In this research, 'Natural' or periodic interpolating cubic spline curve is selected to implement the heuristic path planning. The natural cubic spline curve passes through the given sequence points $(:, j), j=1$ :end. The parameter $t(j)$ for the $\mathrm{j}^{\text {th }}$ point is chosen by Eugene Lee's centripetal scheme, i.e., as accumulated square root of chord length;

$$
\sum_{\mathbf{i}<j} \sqrt{\| \text { points }(:, \mathbf{i}+\mathbf{1})-\text { point }(:, \mathbf{i}) \|_{2}}
$$

if the first and last point coincide (and there are no other repeated points), then a periodic cubic spline curve is constructed. However, double points results in corners.

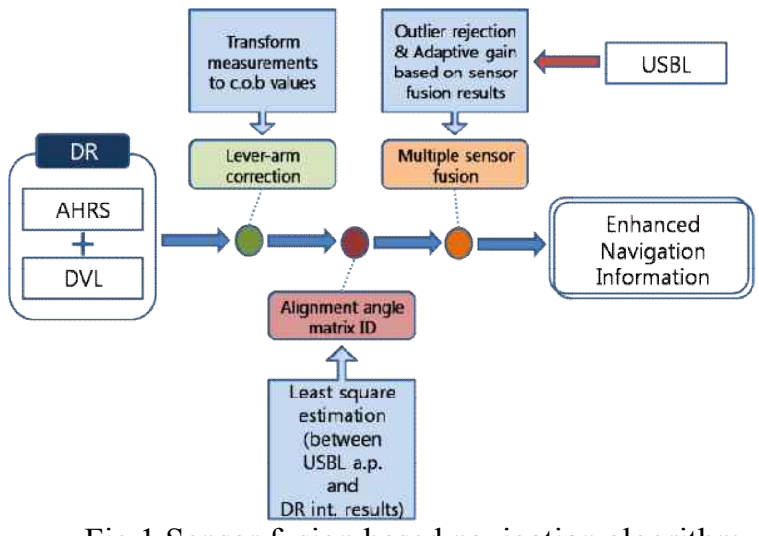

Fig.1 Sensor fusion based navigation algorithm

\section{MULTI-SENSOR FUSION BASED PRECISE UNDERWATER NAVIGATION}

\subsection{Kinematic Relation}

The body-fixed velocity vector $\mathbf{v}_{\mathbf{0}}^{\mathbf{b}}$ are decomposed in the NED reference frame as: (Fossen, 1994)

$$
\dot{\mathbf{p}}^{\mathbf{n}}=\mathbf{R}_{\mathbf{b}}^{\mathbf{n}}(\boldsymbol{\Theta}) \mathbf{v}_{\mathbf{o}}^{\mathbf{b}}
$$

where $\dot{\mathbf{p}}^{\mathbf{n}}$ in the NED velocity vector.

Expanding (1) yields:

$$
\begin{gathered}
\dot{\mathrm{n}}=\mathrm{u} \cos \psi \cos \theta+\mathrm{v}(\cos \psi \sin \theta \sin -\sin \psi \cos \phi) \\
+\mathrm{w}(\sin \psi \sin \phi+\cos \psi \cos \phi \sin \theta) \\
\dot{\mathrm{e}}=\mathrm{usin} \psi \cos \theta+\mathrm{v}(\cos \psi \cos \phi+\sin \phi \sin \theta \sin \psi) \\
+w(\sin \theta \sin \psi \cos \phi-\cos \psi \sin \phi)
\end{gathered}
$$

Based on the assumption that $\square$ and $\boldsymbol{\theta}$ are small, Eq. (3), (4) can be rewritten as follows:

$$
\begin{aligned}
& \dot{\mathbf{n}}=\mathbf{u} \cos \psi-\mathrm{vsi} \psi \\
& \dot{\mathrm{e}}=\mathrm{u} \sin \psi+\mathrm{v} \cos \psi
\end{aligned}
$$

where $u$ and $v$ are velocities measured at body fixed point o. And $\dot{\mathrm{n}}$ and $\dot{\mathrm{e}}$ are velocities in navigation frame.

\subsection{Precise Heading angle alignment}

To implement a precise DR based navigation algorithm, the heading alignment angle errors should be calibrated correctly to minimize cumulative global DR based navigation error.

\subsection{Multi-Sensor Fusion Scheme}

A combined estimate $\hat{\mathrm{x}}$ with error variance matrix $\mathrm{P}$ can be estimated starting with two observable estimates $\hat{\mathrm{x}}_{1}$ and $\hat{\mathrm{x}}_{2}$ of the true state $\mathrm{x}$ with corresponding positive definite error variance matrices $\mathrm{P}_{1}$ and $\mathrm{P}_{2}$. For uncorrelated estimation errors of MMSE(minimum mean square error) estimates $\hat{\mathrm{x}}_{1}$ and $\hat{\mathrm{x}}_{2}$, the overall MMSE estimate is given by basic convex combination of the two estimates (Fränken, 2005),

$$
\begin{gathered}
\hat{\mathbf{x}}=\mathbf{P P}_{1}^{-1} \hat{\mathbf{x}}_{1}+\mathbf{P P}_{2}^{-1} \hat{\mathbf{x}}_{2} \\
\mathbf{P}^{-1}=\mathbf{P}_{1}^{-1}+\mathbf{P}_{2}^{-1}
\end{gathered}
$$

It is well known that in presence of correlation between the two initial estimation errors, the estimated $\mathrm{P}$ may become far too optimistic and that this can finally cause divergence in sequential filtering.

At least a conservative estimate can be given without any detailed knowledge about the correlation. By applying the covariance intersection as follows:

$$
\begin{gathered}
\widehat{\mathbf{x}}=\omega_{1} \mathbf{P P}_{1}^{-1} \hat{\mathbf{x}}_{1}+\omega_{2} P P_{2}^{-1} \widehat{\mathbf{x}}_{2} \\
P^{-1}=\omega_{1} P_{1}^{-1}+\omega_{2} P_{2}^{-1}
\end{gathered}
$$

with nonnegative coefficients $\boldsymbol{\omega}_{1}$ and $\boldsymbol{\omega}_{2}$ obeying

$$
\omega_{1}+\omega_{2}=1
$$

Herein, the coefficients $\omega_{1}$ and $\omega_{2}$ are usually chosen as to minimize either the trace or the determinant of $P$.

Finding the solution of a highly nonlinear optimization problem such as Eq.(12) needs high numerical implementation efforts. To avoid this, Niehsen has proposed to use a fast approximate solution instead (Niehsen, 2002). When trace $\left(\mathrm{P}_{1}\right) \ll \operatorname{trace}\left(\mathrm{P}_{2}\right)$, one would expect to get $\omega_{1} \approx 1$ to minimize overall trace.

$$
\omega_{1} \text { trace }\left(P_{1}\right)-\omega_{2} \text { trace }\left(P_{2}\right)=0
$$

that, with (12c), is given by

$$
\omega_{1}=\frac{\operatorname{tace}\left(\mathbf{P}_{2}\right)}{\operatorname{tace}\left(\mathbf{P}_{1}\right)+\operatorname{trace}\left(\mathbf{P}_{2}\right)}
$$

where $\mathbf{0}<\boldsymbol{\omega}_{1}<\mathbf{1}$.

In Eq. (14), trace can be replaced by determinant by preference instead.

$$
\omega_{1}=\frac{\operatorname{det}\left(P_{2}\right)}{\operatorname{det}\left(P_{1}\right)+\operatorname{det}\left(P_{2}\right)}
$$

In this paper, authors propose a new scheme which has a receding horizon strategy to cope with changing sensor status as follows:

$$
\begin{aligned}
& \hat{\mathbf{x}}= \omega_{1 \mathrm{k}} \mathbf{P}_{\mathbf{k}} \mathbf{P}_{1 \mathrm{k}}^{-1} \hat{\mathbf{x}}_{1}+\omega_{2 \mathrm{k}} \mathbf{P}_{\mathbf{k}} \mathbf{P}_{2 \mathrm{k}}^{-1} \hat{\mathbf{x}}_{2} \\
& \mathbf{P}_{\mathrm{k}}^{-1}=\omega_{1 \mathrm{k}} \mathbf{P}_{1 \mathrm{k}}^{-1}+\omega_{2 k} \mathbf{P}_{2 \mathrm{k}}^{-1}
\end{aligned}
$$


with nonnegative coefficients $\boldsymbol{\omega}_{\mathbf{1 k}}$ and $\boldsymbol{\omega}_{\mathbf{2 k}}$ obeying

$$
\omega_{1 \mathrm{k}}+\omega_{2 \mathrm{k}}=1
$$

where subscript $\mathrm{k}$ means which value determined with design data span (computed with $n$ data). For example, $\mathbf{P}_{\mathbf{k}}$ can be calculated as $\sum_{\mathbf{i}=\mathbf{k}-\mathbf{n}+\mathbf{1}}^{\mathbf{k}} \mathbf{P}(\mathbf{i})$.

In this research, estimates $\hat{\mathrm{x}}_{1}$ can be a local dead-reckoning estimate and $\hat{\mathrm{x}}_{2}$ can be an outlier rejected USBL measurement for the navigation solution $\hat{\mathrm{x}}$.

\subsection{Outlier Rejection Scheme}

In this study, an intuitive outlier rejection algorithm is proposed. The basic idea is from the robust local regression method (Cleveland, 1979). Cleveland's algorithm can be applied to USBL positioning effectively to remove outliers. But calculation time is a burden due to iterative scheme structure.

The proposed scheme for robust weights is given by the simple bisquare function shown below.

$$
\omega_{i}=\left\{\begin{array}{cc}
1 & \left|r_{i}\right|<3 \gamma \\
0 & \left|r_{i}\right| \geq 3 \gamma
\end{array}\right.
$$

$\mathbf{r}_{\mathbf{i}}$ is the residual of the ith USBL data point produced by the multi-sensor fusion results, and $\boldsymbol{\gamma}$ is the RMS error which can be measured during USBL calibration, for example, $0.3 \%$ of slant range. The number 3 in front of $\boldsymbol{\gamma}$ is design factor. Authors selected the smaller value compared with original Cleveland's scheme.

While Cleveland's robust weights function is the distribution function for some data span, author's weights are designed for selecting or not as a new data.

\section{Path generation Simulation}

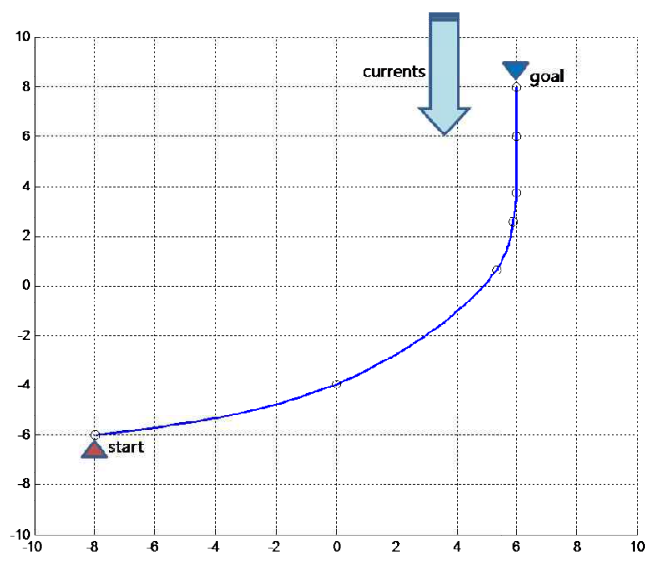

Fig.2 Path generation example for NS currents

Fig. 2 shows path generation simulation results for north-south currents exist where nodal points are selected by an experienced human supervisor. The way-point should be selected as the final approach angle is against the major current axis.

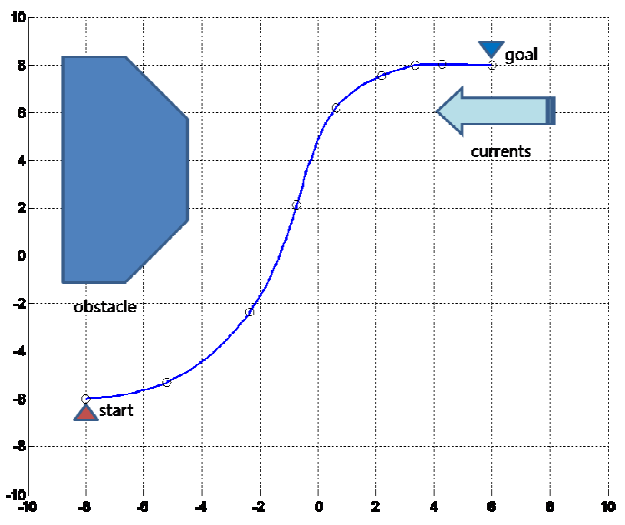

Fig.3 Path generation example for EW currents and terrain obstacles

Fig. 3 shows that path generation results currents are from east to west with terrain obstacles where the underwater vehicle cannot go. More complex condition can be handled by human supervised waypoints selection. And the piecewise natural cubic spline scheme can generate a smooth path for underwater vehicle to be controlled well.

\section{MULTI-SENSOR FUSION BASED NAVIGATION ALGORITHM RESULTS AND DISCUSSIONS}

Table 1 Specifications of sensors

\begin{tabular}{|c|c|}
\hline Sensors & Specifications \\
\hline \multirow{2}{*}{ FOG } & Heading Accuracy: $0.1 \mathrm{deg}$ \\
AHRS & Roll, Pitch Accuracy: $0.01 \mathrm{deg}$ \\
& Update rate $: 100 \mathrm{~Hz}$ \\
\hline \multirow{2}{*}{ USBL } & Freq.: $7.5-18 \mathrm{kHz}$ \\
& Accuracy : $0.3 \%$ of slant range \\
& Update periods $: 4 \sim 8 \mathrm{sec}$. \\
\hline \multirow{2}{*}{ DVL } & Freq.: $1,200 \mathrm{kHz}$, \\
& Bottom lock range: $0.5 \mathrm{~m} \sim 30 \mathrm{~m}$ \\
& Accuracy: $+/-0.2 \%$ \\
\hline
\end{tabular}

Table 1 shows specifications of sensors used for the precise navigation algorithm verification.

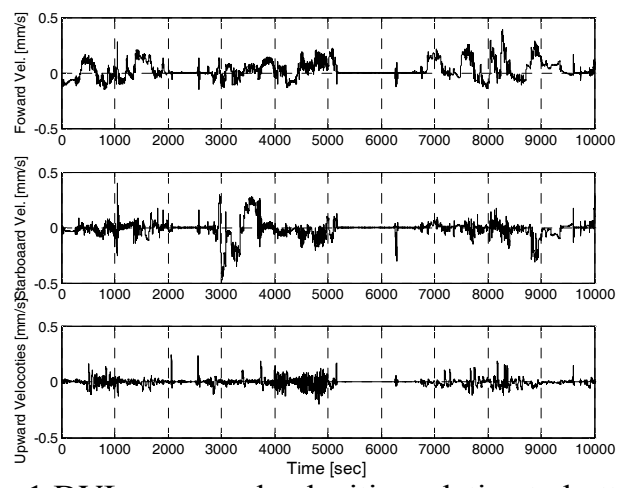

Fig. 1 DVL measured velocities relative to bottom 

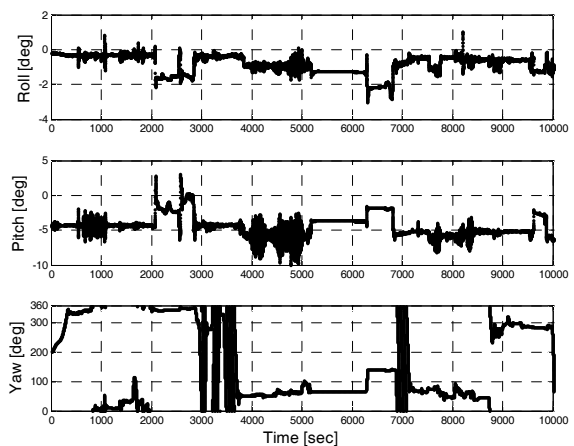

Fig. 2 IMU measured attitudes during exploration

Fig. 4 and 5 depicts measured sensor values of DVL and IMU during the East-sea exploration for the survey on the $1,500 \mathrm{~m}$ depth gas seeping area which is located at the latitude of $36^{\circ} 05.88^{\prime} \mathrm{N}$ and the longitude of $130^{\circ} 04.73^{\prime} \mathrm{E}$. We can observe that offsets from mean values are small enough to satisfy the former assumption for $2 \mathrm{D}$ kinematic relation.

Fig. 6-8 shows outlier rejection algorithm results of USBL positioning data (initial position: $3605.850 \mathrm{~N}$, $13004.685 \mathrm{E}$ ). The outliers are not so frequent in normal conditions, while the outliers observed here are mainly due to the ROV was in a methane hydrate area. Results show that outliers are removed effectively. These results are generated by a concurrent MSF and outlier rejection algorithm. The outlier rejection rate for this data set is $6.65 \%$.

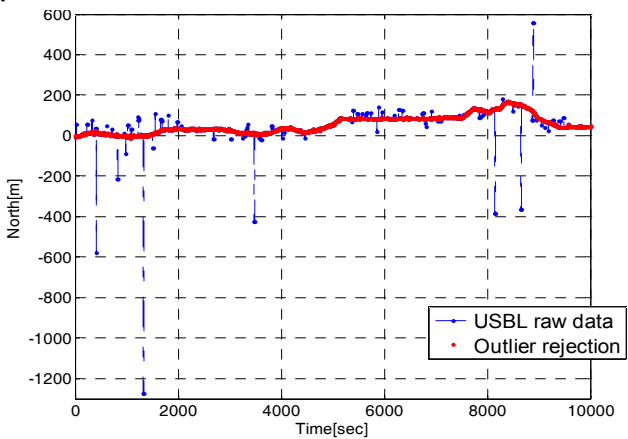

Fig. 3 Outlier rejection algorithm results for North $[\mathrm{m}]$ coordinate

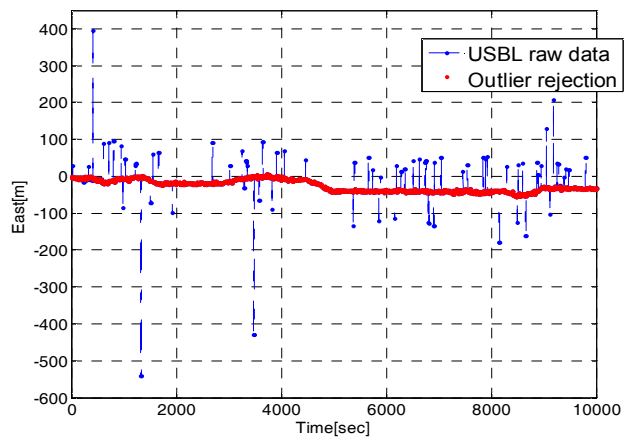

Fig. 4 Outlier rejection algorithm results for East $[\mathrm{m}]$ coordinate

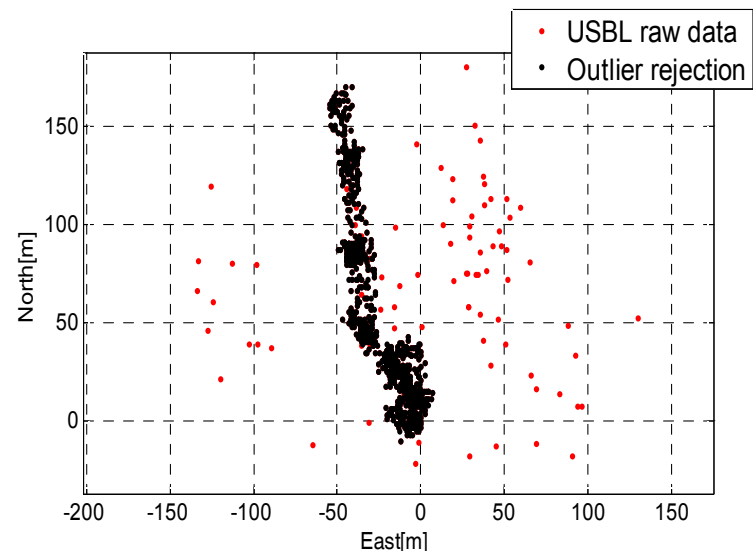

Fig. 5 Outlier rejection algorithm results in 2D navigation frame

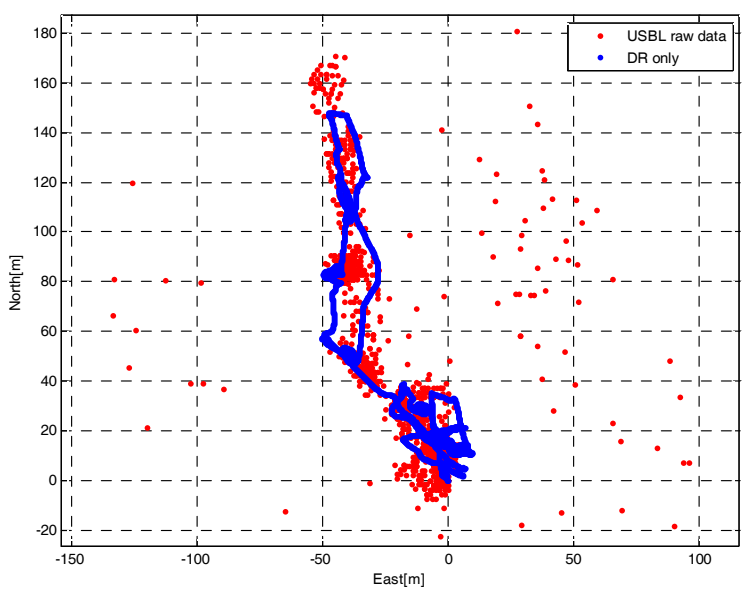

Fig. 6 Standalone dead reckoning results after heading alignment calibration

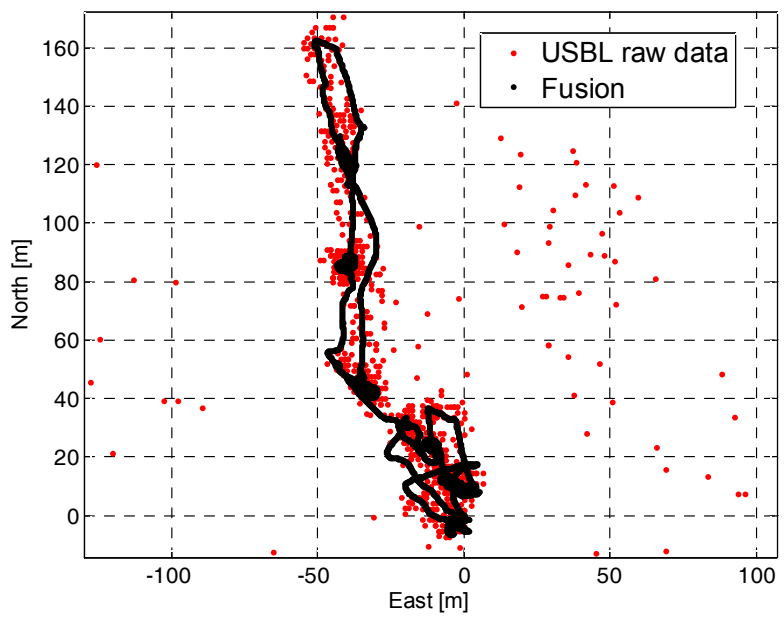

Fig. 7 MSF based estimation results with sea trial data 

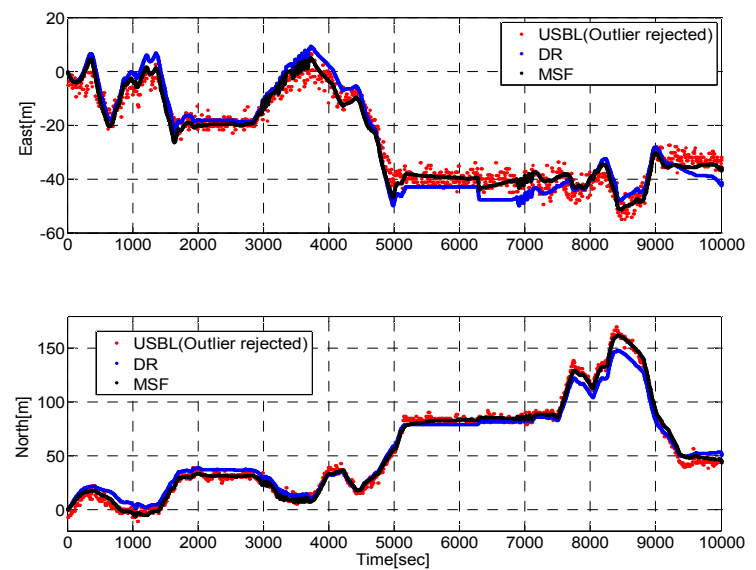

Fig. 8 Comparison between USBL (Outlier rejected), DR and MSF results
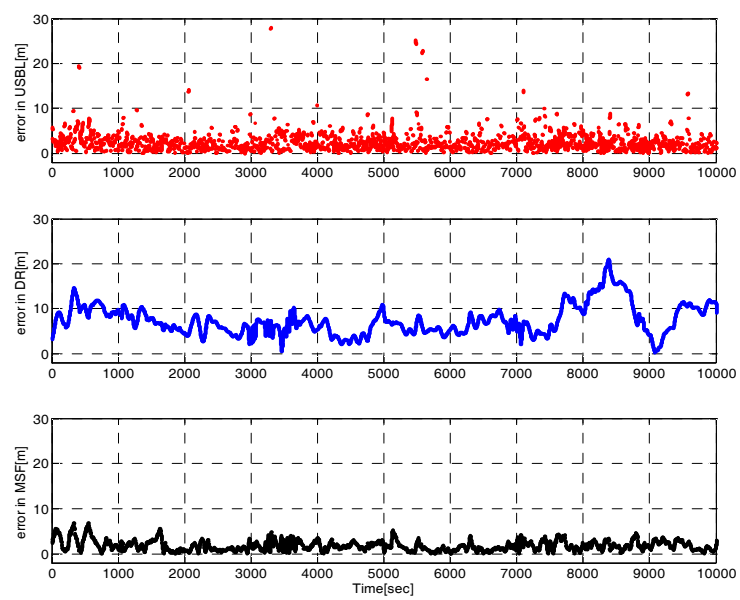

Fig. 9 Error bounds of standalone DR and MSF algorithm

Fig. 9 shows standalone dead reckoning results after heading alignment compensation in the navigation frame. Because the data was collected in a wide area and the track has a special feature, the constant heading alignment error can be identified by general least square method. The alignment error sources are mainly due to position offset (lever arm) and angle difference (calibration angle) between the body frame axis and the sensor frame axis. Still, accumulative errors due to scale error, dynamic attitude estimation error and numerical integration error are present. To prevent this error accumulation, the outlier rejected USBL data can be used for accuracy enhancement. Fig. 10 depicts the MSF based navigation algorithm performance in this research. Results shows that MSF algorithm generates precise estimates with good accuracies and $5 \mathrm{~Hz}$ update rate, which merges the merits of DR (DVL+IMU) and USBL.

Fig. 11 compares outlier removed USBL data, standalone DR and MSF algorithm result in East and North coordinates. MSF algorithm results follows outlier rejected USBL data with good accuracy, while standalone DR result has some errors. Fig. 12 depicts the errors of USBL,
DR and MSF algorithm against smoothed USBL data (Cleveland's scheme) respectively. While the standalone DR scheme shows larger error and drift with time, MSF algorithm results shows smaller and bounded error.

\section{SUMMARY}

In this paper, practical path generation scheme considering final approach angle due to ocean currents and a precise multi-sensor fusion based underwater navigation algorithm are designed.

Authors introduced natural cubic spline curve generation scheme which has a good performance for corner treatment. Human recognition can be introduced by way-points selection during the path generation procedure.

Authors also investigated kinematic relations for the precise dead reckoning algorithm. In addition the error sources of standalone DR algorithm are sought. From the kinematic relation and calculation results, we can conclude that the heading alignment angle should be identified and compensated with in-situ measurement data. The practical MSF algorithm for precise and accurate navigation in the horizontal navigation frame is designed and verified. This algorithm is also robust to outliers and easy to implement as a real time navigation solution. To demonstrate the performance of the designed MSF based navigation algorithm, software in the loop simulation with experiment data is carried out.

\section{ACKNOWLEDGEMENT}

This work was done as a part of the research projects, "Development of a Next Generation Deep-Sea Unmanned Underwater Vehicle", which are financially supported by the Ministry of Land, Transport and Maritime Affairs (MLTM) of Republic of Korea and "Development of technologies for an underwater robot based on artificial intelligence for highly sophisticated missions", which is supported by KORDI.

\section{REFERENCES}

[1] E.T.Y Lee, "Choosing nodes in parametric curve interpolation, Computer-Aided Design 21, pp. 363-370, 1989.

[2] L. Whitcomb, D. Yoerger and H. Singh, "Advances in Doppler-Based Navigation of Underwater Robotic Vehicles," Proc. Of IEEE Int. Conf. on Robotics \& Automation, pp. 399-406, May, 1999.

[3] J.C. Kinsey and L.L. Whitcomb, "In Situ Alignment Calibration of Attitude and Doppler Sensors for Precision Underwater Vehicle Navigation: Theory and Experiment," IEEE Journal of Oceanic Engineering, Vol. 32, No. 2, pp. 286-299, 2007. 
[4] D. Fränken and A. Hüpper, "Improved Fast Covariance Intersection for Distributed Data Fusion," Int. Conf. on Information Fusion, pp. 154-160, 2005.

[5] W.S. Cleveland, "Robust Locally Weighted Regression and Smoothing Scatter plots," Journal of the American Statistical Association, Vol. 74, pp. 829-836, 1979
[6] T. I. Fossen, Guidance and control of ocean vehicles, John Wiley \& Sons, 1994

[7] W. Niehsen, "Information fusion based on fast covariance intersection filtering," In Proc. Int. Conf: Inf: Fusion (FUSION '02), pages 901-905, July, 2002. 